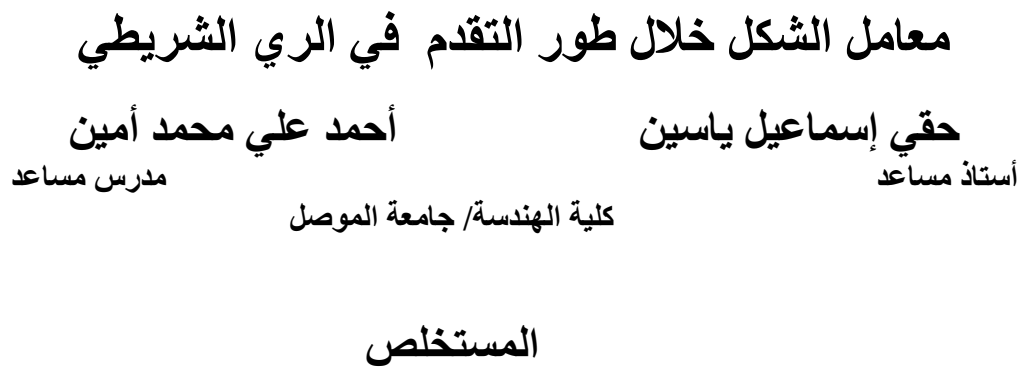

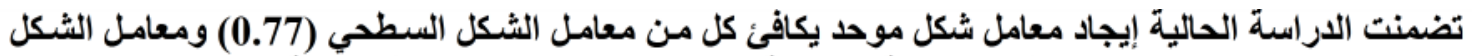

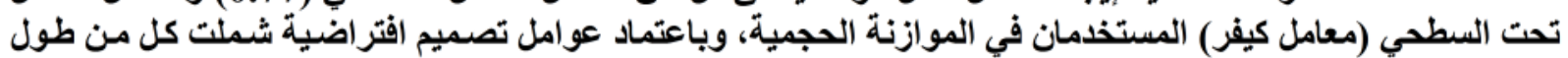

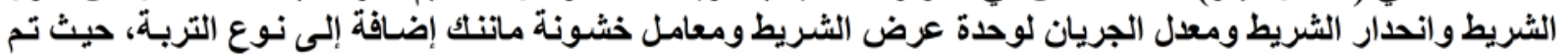

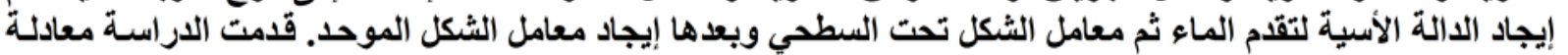

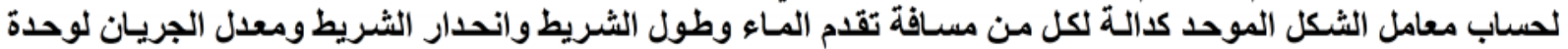

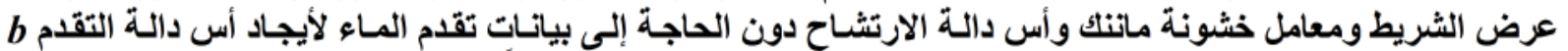

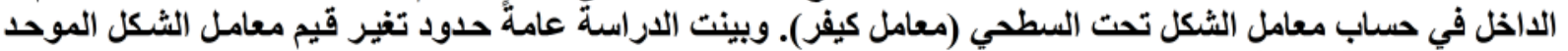
الشريط

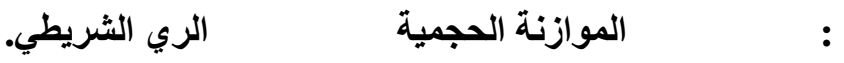

\title{
Shape Factor During Advance Phase in Border Irrigation
}

\begin{abstract}
The present study proposed a new single shape factor to be used in volumetric balance equation. The new single shape factor combines the surface shape factor $(0.77)$ and the subsurface shape factor (Kiefer factor) in one shape factor. By depending on hypothetical design factors: border length, border slope, inflow rate per unit width of border, manning coefficient, as well as soil type, the power function of water advance, the subsurface shape factor and single shape factor have been found respectively. The study introduced an equation to calculate the single shape factor as a function of water advance distance, border length, border slope, inflow rate per unit width of border , manning coefficient and the exponent of infiltration function without needing to data for water advance or the exponent of advance function $(b)$, which is used to calculate the subsurface shape factor (Kiefer factor). Generally, the study demonstrated that the variation limits of the values of the single shape factor are (0.73-0.76) with average of 0.75 when the ratio of advance distance to total border length greater than or equal to 0.4.
\end{abstract}

Key words: shape factor, volume balance, advance phase, border irrigation.

$11-11-2013:$

$14-1$ - 2013 : 


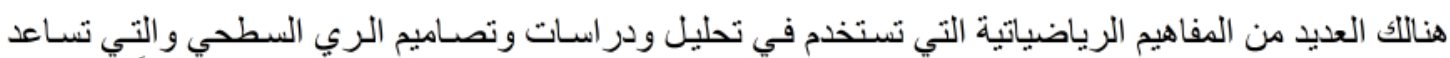

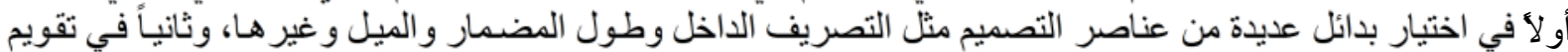

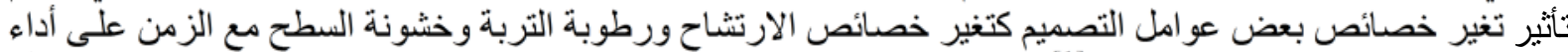

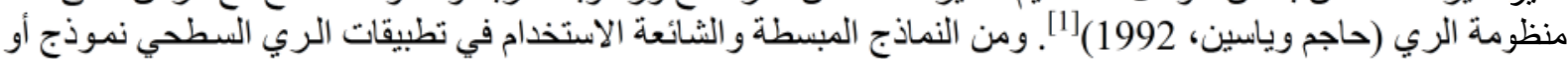

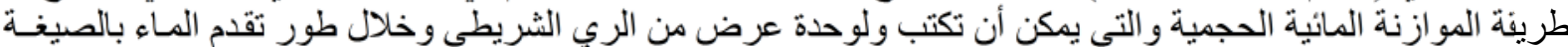
الآتية(Christiansen et al. 1966)

$q * t_{x}=\sigma_{y} * d_{\circ} * x+\sigma_{z} * D_{\circ} * x$

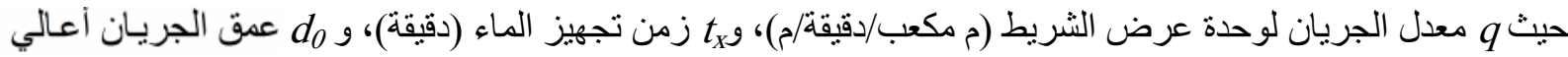
مضمار الري (م)، وX مسافة تقدم الماء عند الزمن الثكل السطحي و الذي يمثل نسبة معدل عمق الجريـان على امتداد مسـافة تقدم المـاء X

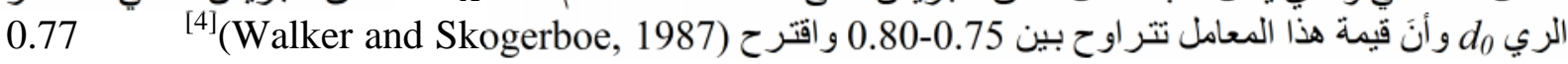
O معامل الشكل تحت السطح والذي يمثل نسبة معدل عمق الارتشاح على امتداد مسافة تقدم

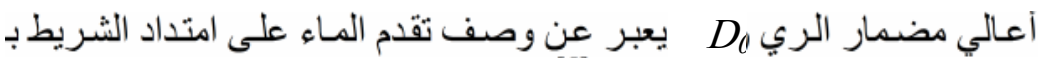

$$
\begin{aligned}
& \text { : }{ }^{[5]} \text { (Fok and Bishop, 1965) }
\end{aligned}
$$

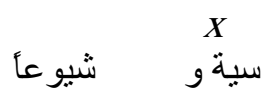

$$
x=a t_{X}^{b}
$$

ي التربة بدالة كوستاكوف الآتية:

$D=c t^{m}$

(دقيقة) و و وm ثوابت وضعية. و عندما t في المعادلة

D في المعادلة (3) يسـاوي Do في المعادلة (1). ويعتمد معامل الشكل تحت السطح

Elliot and Walker, ) ${ }^{[5]}$ (Fok and Bishop, 1965) $b$ : (Kiefer) (Walker and Skogerbe, 1987)

$\sigma_{Z}=(1+b+m-b m) /(1+b+m+b m)$

لقد تم تطبيق طريقة الموازنة المائية الحجمية في الري السطحي من قبل العديد من الباحثين لغرض در استة طور تقدم المـاء

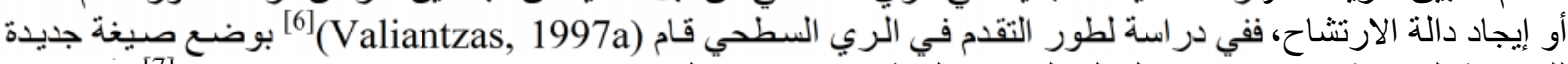

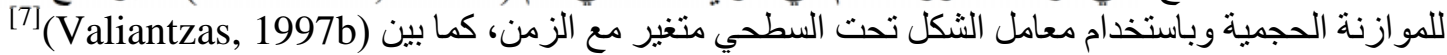

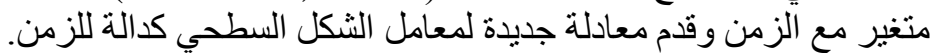

(8lazba, 1999)

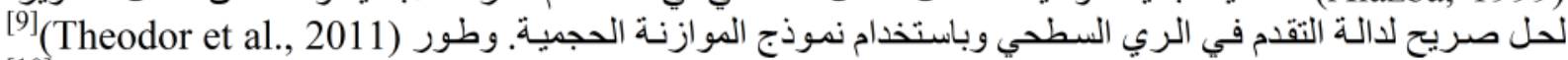

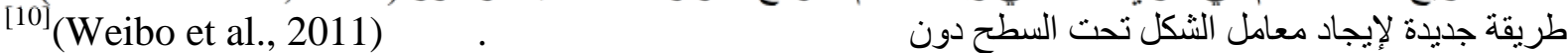

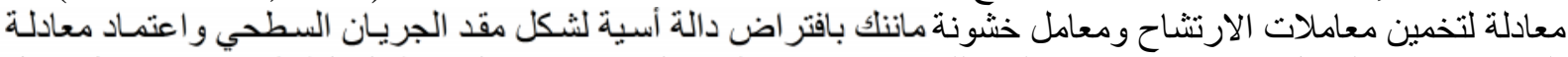

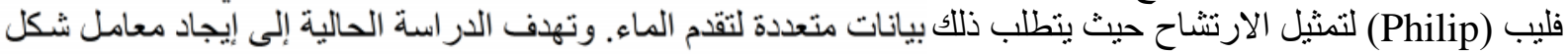

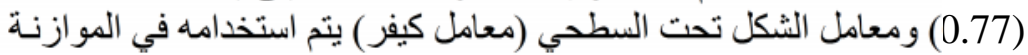

$$
\text { الحجمية، حيث تصبح المعادلة (1) بالصيغة الآتية وبمعامل شكل موحد }
$$

$q^{*} t_{x}=\sigma^{*} X^{*}\left(d_{0}+D_{0}\right)$ 
تم اعتماد عو امل تصميم افتر اضية واسعة تشمل كل من طول الثريطو انحدار الثريط ومعدل الجريـان لوحدة

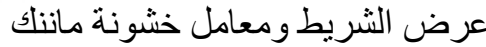

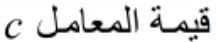

3125 حالة تداخل للقيم
(3)

$$
\begin{aligned}
& \text { m بتصنيف يعتمد معدل الارتشاح }
\end{aligned}
$$

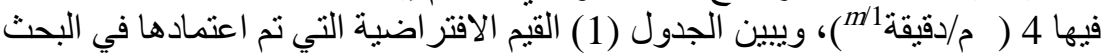

(1): القيم الافتر اضية لعو امل التصميم المعتمدة

\begin{tabular}{|c|c|c|c|c|c|}
\hline 400 & 320 & 240 & 160 & 80 & أطوال الثــريط (م) \\
\hline 2.0 & 1.55 & 1.1 & 0.65 & 0.2 & انحدارات الشـريط (\%) \\
\hline 0.9 & 0.72 & 0.54 & 0.36 & 0.18 & معدلات الجريان (م/دقيقة/م) \\
\hline "0.24 & 0.20 & 0.16 & 0.12 & 0.08 & $(3 / 1 /)$ \\
\hline 16 & 13 & 10 & 7.0 & 4.0 & $(1)$ \\
\hline 0.599 & 0.574 & 0.543 & 0.501 & 0.438 & \\
\hline
\end{tabular}

TII) (حاجم وياسين، 1992)*

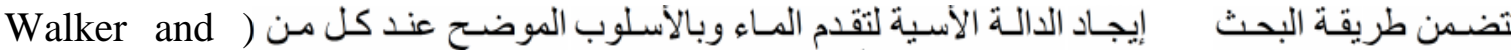

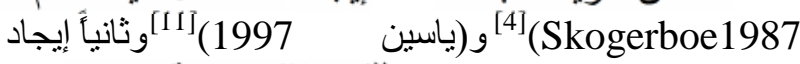
(\%) (\%)
( ) الثريط 1 ( )
للقبم الافتر اضية وتحديد

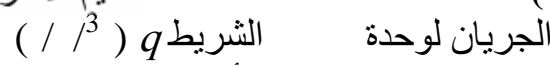

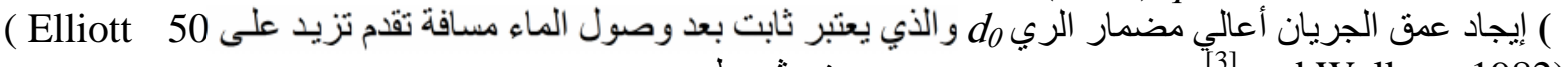

$$
\text { [3] [3 and Walker 1982): }
$$

$$
d_{0}=\left[n^{*} q\right]^{0.6} / s^{0.3}
$$

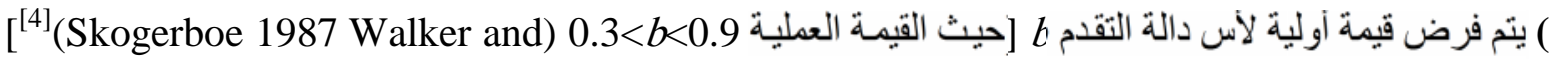

$$
\text { ( ) } \sigma_{z}
$$

إيجاد

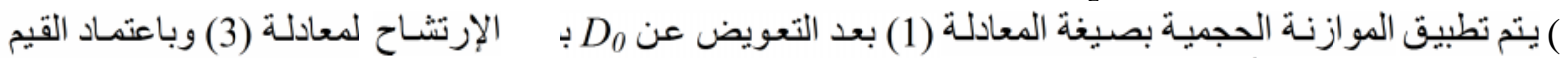
الإفتر اضية المحدة في () t/2 الإيجاد

$$
\begin{aligned}
& q^{*} t_{1}=\sigma_{y}{ }^{*} d_{0} * 1+\sigma_{z}^{*} c^{*} t_{1}^{m *} 1 \ldots \ldots \ldots \ldots \\
& q^{*} t_{1 / 2}=\sigma_{y}{ }^{*} d_{0} * 1 / 2+\sigma_{z}{ }^{*} c^{*} t_{1 / 2}^{m} * 1 / 2
\end{aligned}
$$

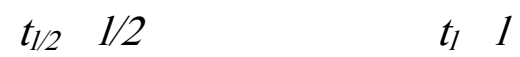

$b$

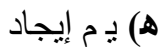

$b=\ln 2 / \ln \left[t_{1} / t_{1 / 2}\right]$

حيث يتم تغيير القيمة المفروضـة وا b المفروضـة وطبقاً للاقة المحددة. ه $\quad b \quad t_{1 / 2} t_{1}$
) يتم المقارنة بين قيمة b $\quad$ ل bـ ${ }^{[12]}$ 2010 Microsoft Excel Solver 
a (2) يتم حسـ ثابت دالة التقدم a

t

) بعد إيجاد آمثل قيمـة لأس

$a=1 / t_{1}^{b}$

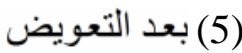

$$
\begin{array}{llll}
1 \ldots . . & 70 & 60 & 50
\end{array}
$$

) يتم إيجاد

$D_{0}$

$\sigma=q^{*}(x / a)^{1 / b} /\left[X^{*}\left(d_{0}+c^{*}\left\{(x / a)^{1 / b}\right\}^{m}\right)\right]$

تداخل للقيم الافتر اضية لمعاملات التصميم طول الثريط 1

62500

(م) و انحدار الثريطS(\%)

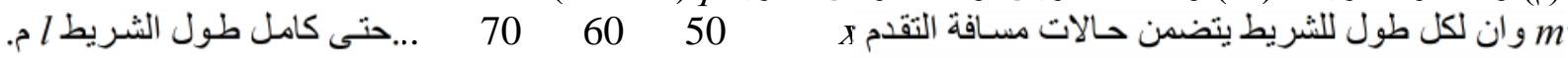

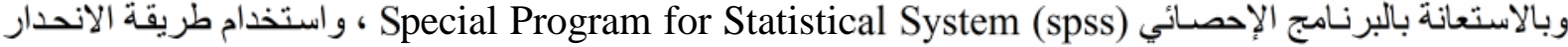

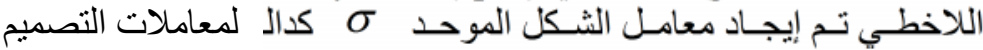

$\sigma=\frac{\left[1.4676(n q)^{0.6} / s^{0.3}-0.0214 I^{0.5249} m^{5.2235} \phi^{4.0612}\right]}{\left[1.9351(n q)^{0.6} / s^{0.3}+0.1095 \phi^{-1.5736} m^{4.7107}\right]}$.

حيث ه تمثل نسبة مسافة التقدم x إلى طول الثريط الكلي ، بهذه المعدلة يمكن ايجاد معامل الثكل الموحد لتطبيق

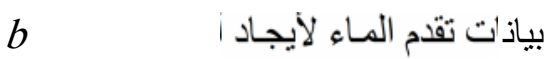

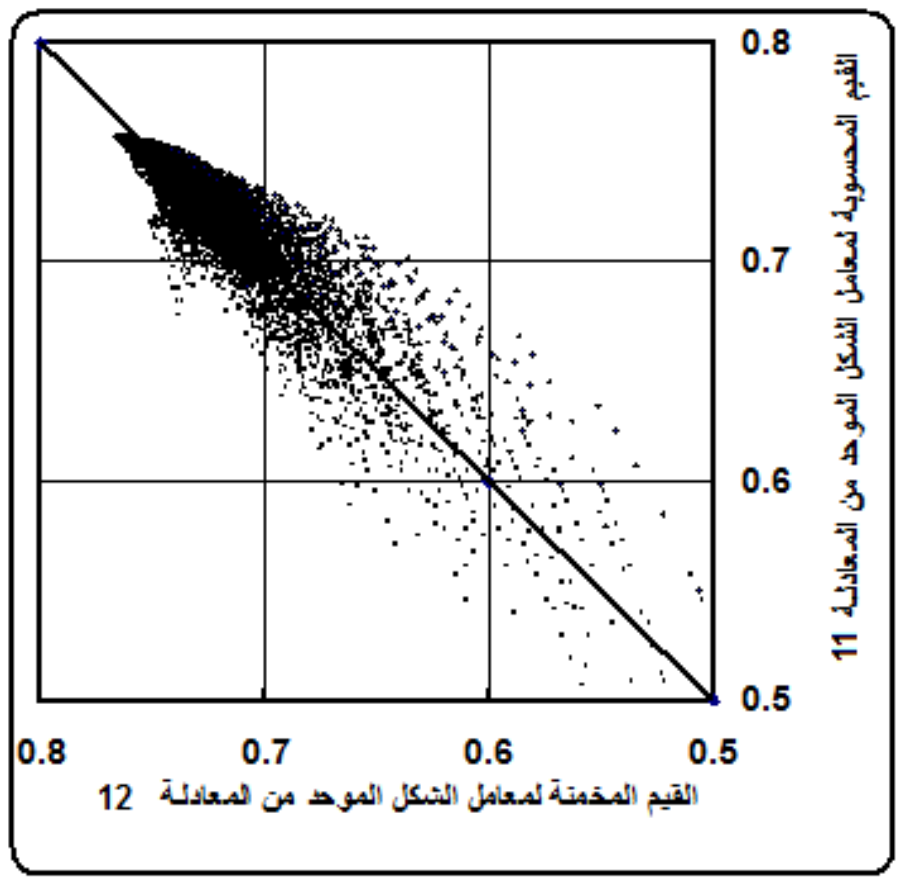

الشكل(1): المقارنة بين القيم المحسوبة

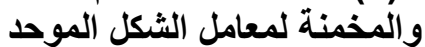

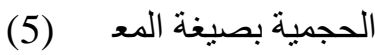

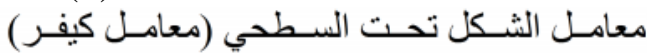

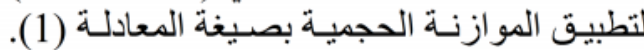

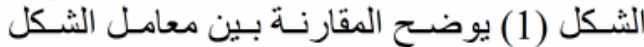
(11)

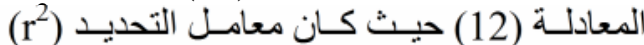
0.886 ويتبين من هذا الثكل عامةً إن اغلب القئ القيم

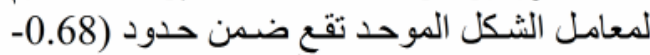
0.75). ولغرض توضيح تأثير معاملات التصنيم التصنيم في المعادلة (12) على ملى معامل الثكل الموحد تبلين

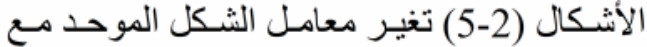
نسبة مسافة التقدم إلى طول الثريط دامل الثريط الكلى وذلك

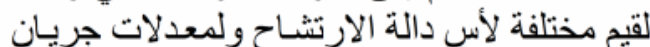

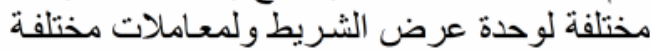

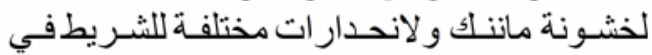
حالة طول الثريط على التو الي، يتضـح من هذانه

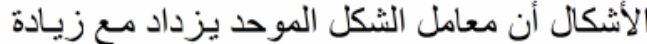

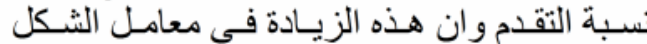

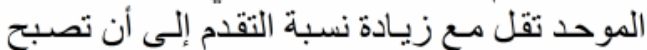

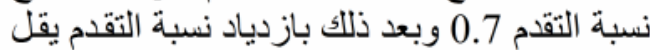

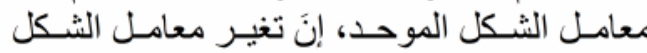

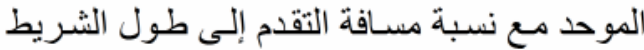

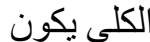




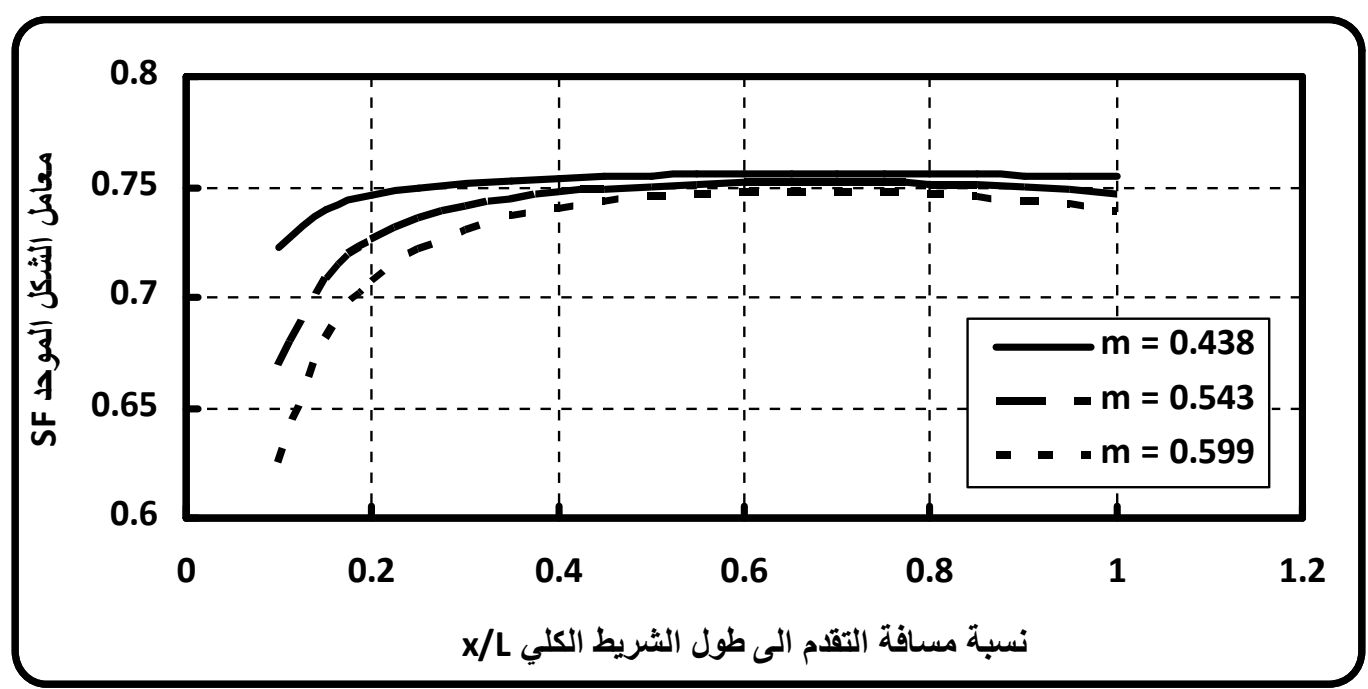

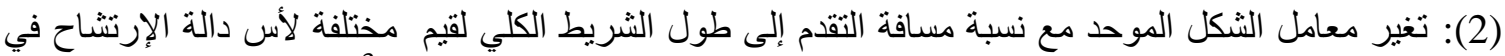

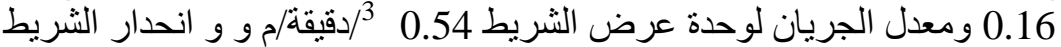

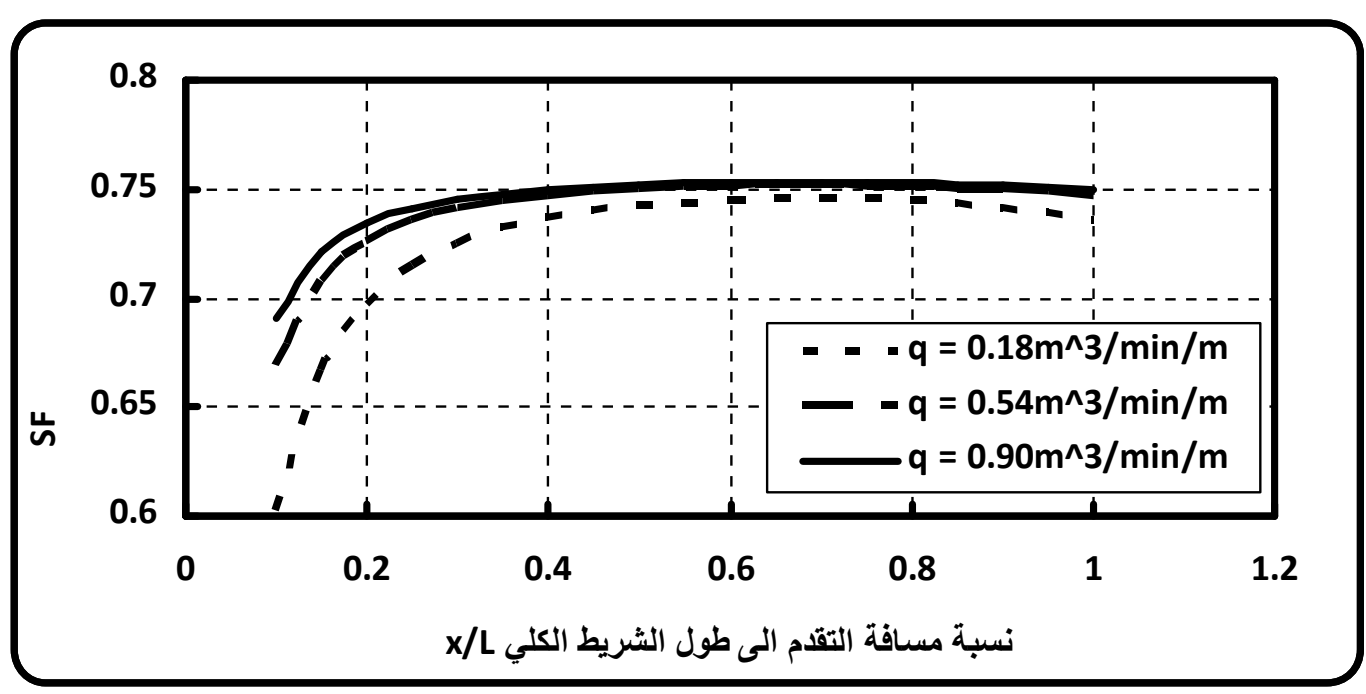

(3): تغير معامل الثكل الموحد مع نسبة مسافة التقام إلى طول الثريط الكلي ولمعدلات جريان مختلفة لوحدة عرض 0.543 


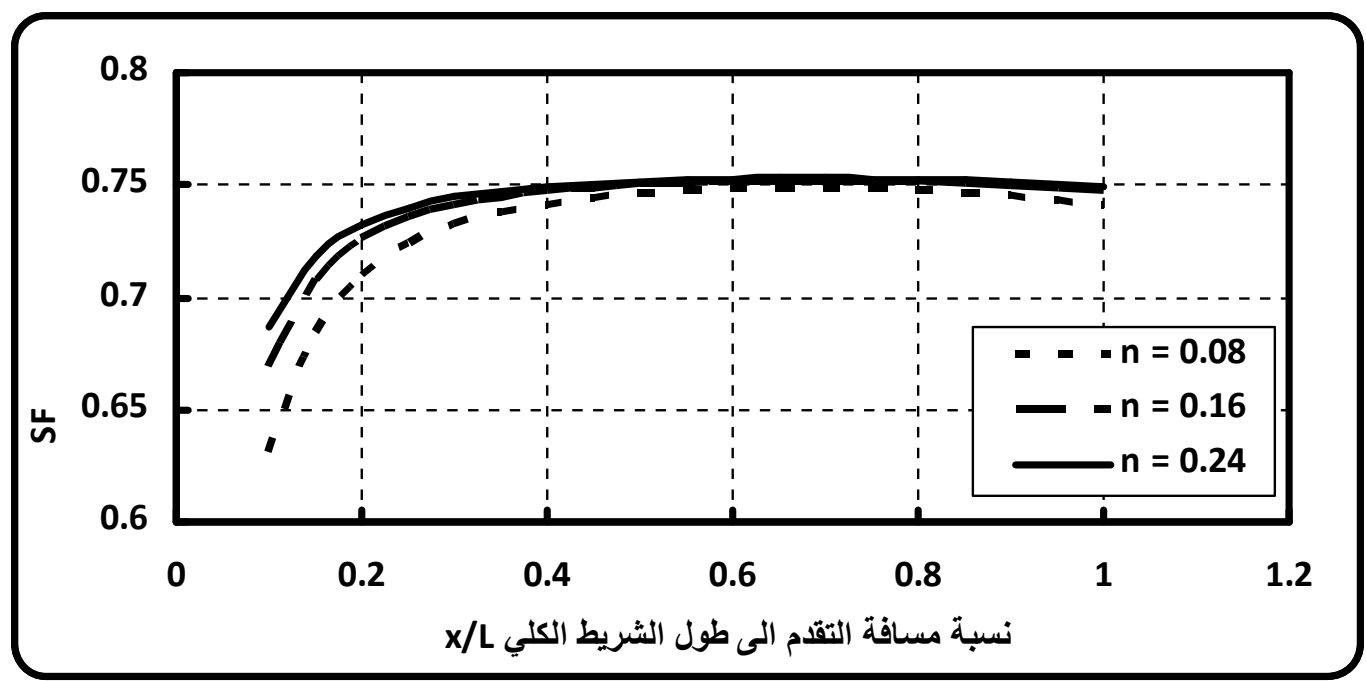

(4): تغير معامل الثكل الموحد مع نسبة مسافة التقدم إلى طول الثريط الكلي لمعاملات مختلفة لخشونة مانتلك في

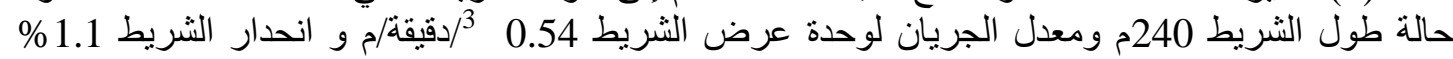
0.543

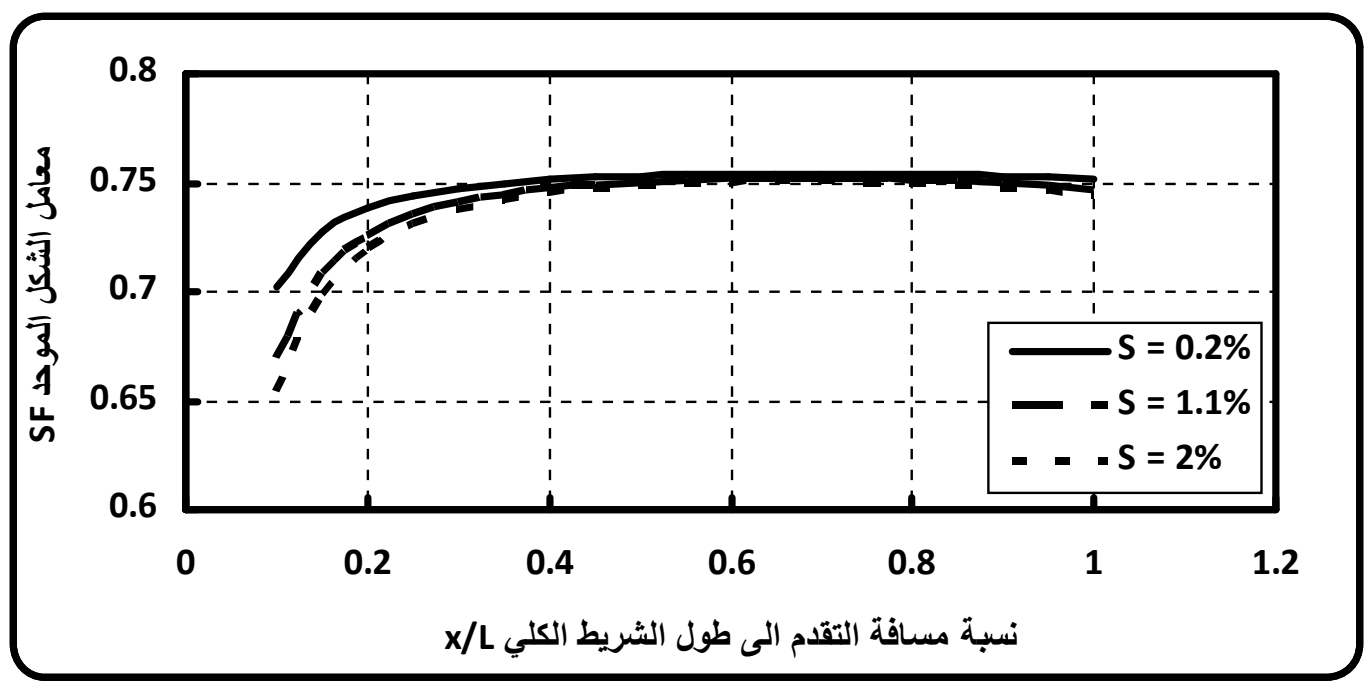

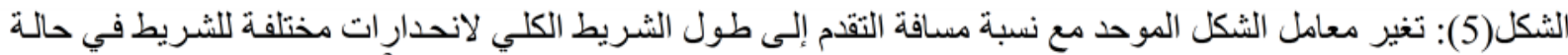

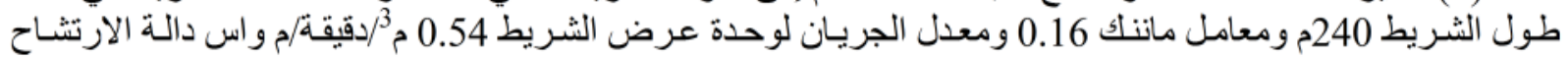

متناظر اللقيم من 0.4 حتى 1 لنسبة التقدم وذلك حول المحور الذي يمر بالقيمة 0.7 لنسبة التقدم، كمـا تبين الآشكال آن

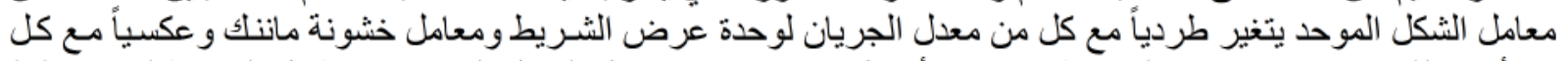

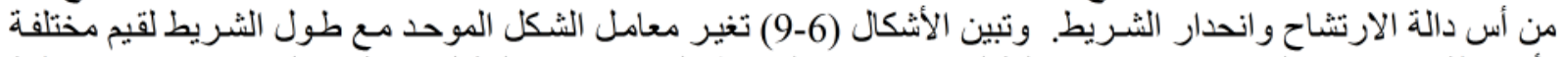

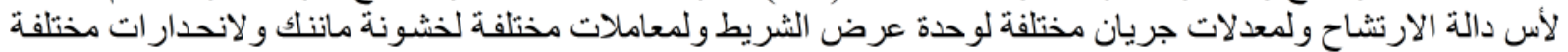

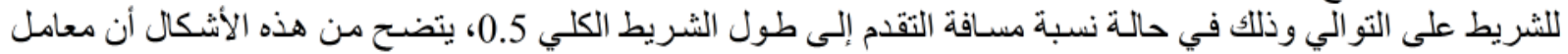

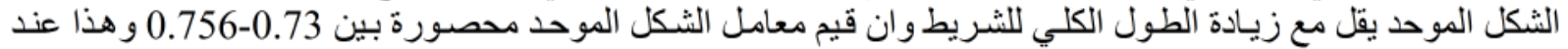

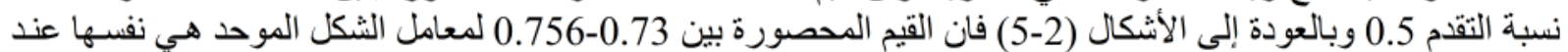
نسبة التقدم 0.9 ومساحة التغير تكون أقل عند نسب التقدام

لك في الحالات التي تكون فيها نسبة مسافة التقدم إلى طول الثريط الكليك 0.4 حيث تبين أن (87\%) 
0.75، ومما تقدم أعلاه فهناك إمكانيـة في التعويض عن قيمـة

قيم معامل الثكل الموحد تتغير من $0.730 .76 \quad$ (5) 0.75

$q^{*} t_{X}=0.75^{*} X^{*}\left(d_{0}+D_{0}\right)$

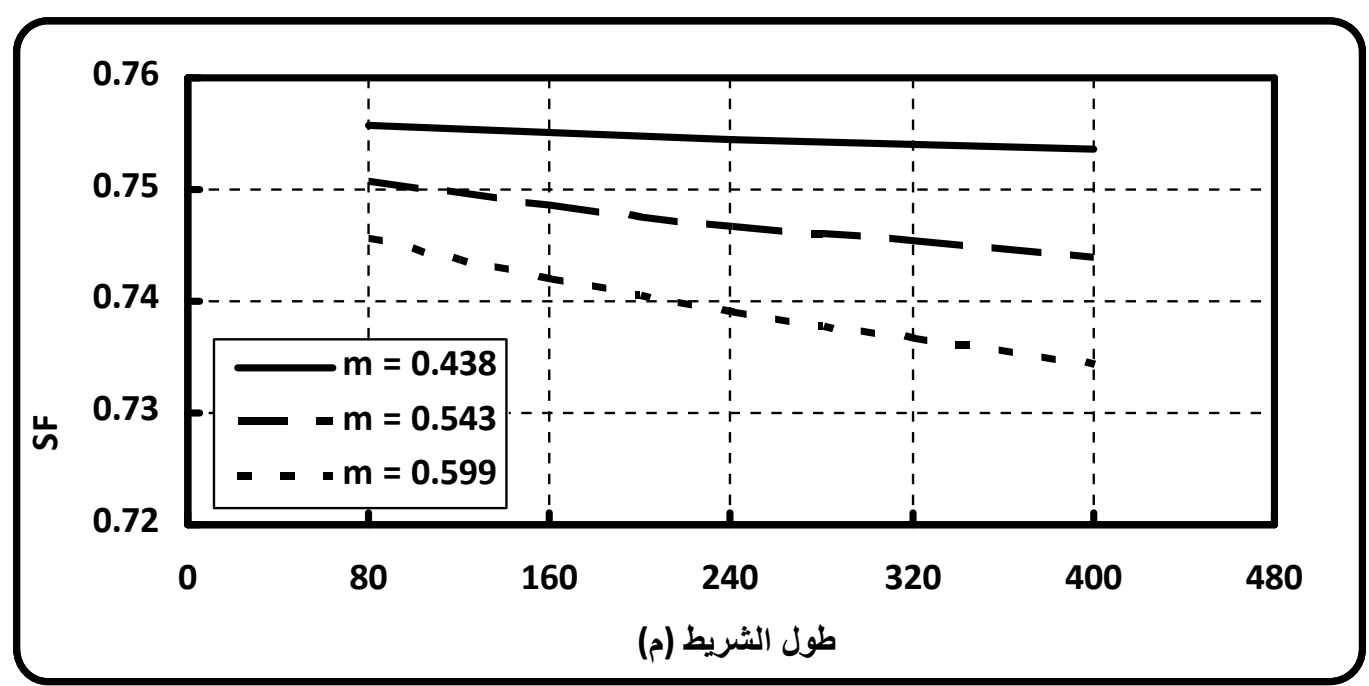

(6): تغير معامل الثكل الموحد مع طول الثريط لقيم مختلفة

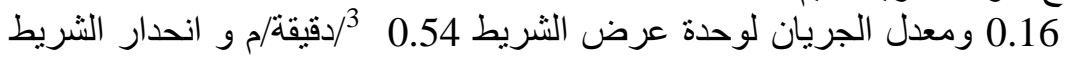

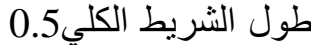
$\% 1.1$

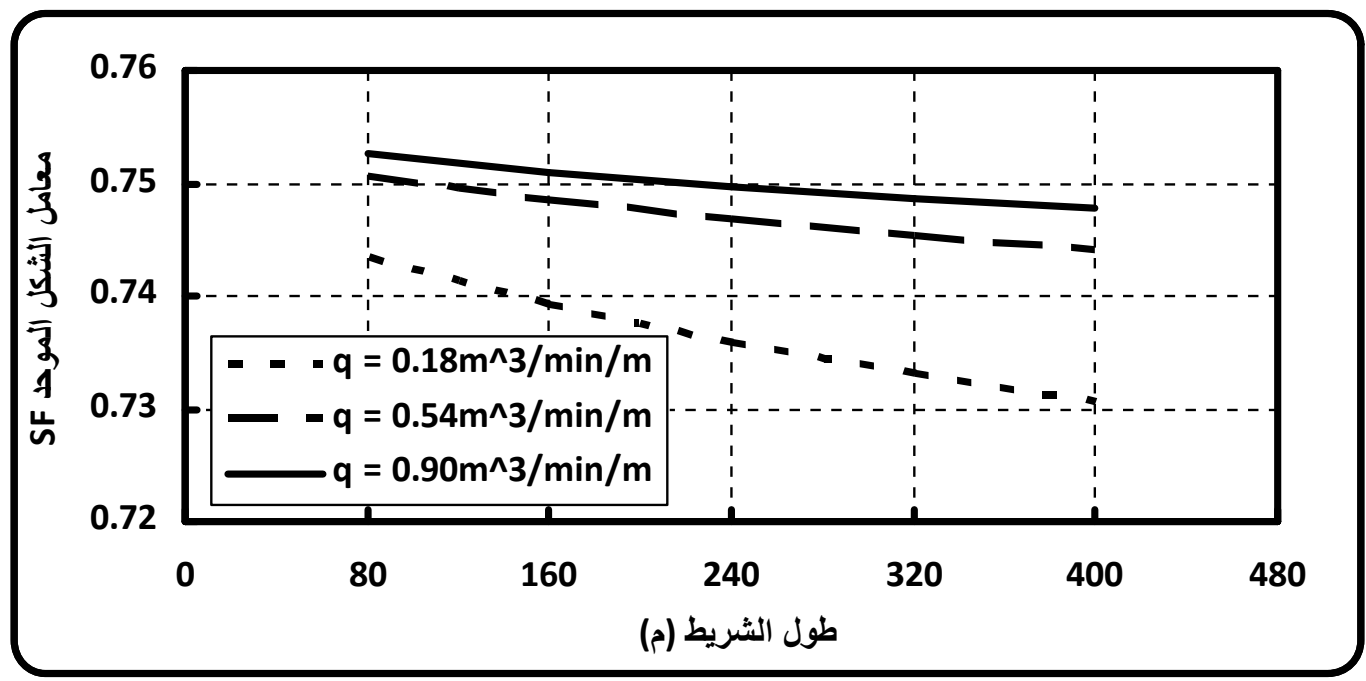

(7): تغير معامل الثكل الموحد مع طول الثريط ولمعدلات جريان مختلفة لوحدة عرض الثريط في حالة نسبة 0.543

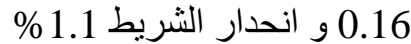

مسافة التقدم إلى طول الثريط الكلي 0.5 


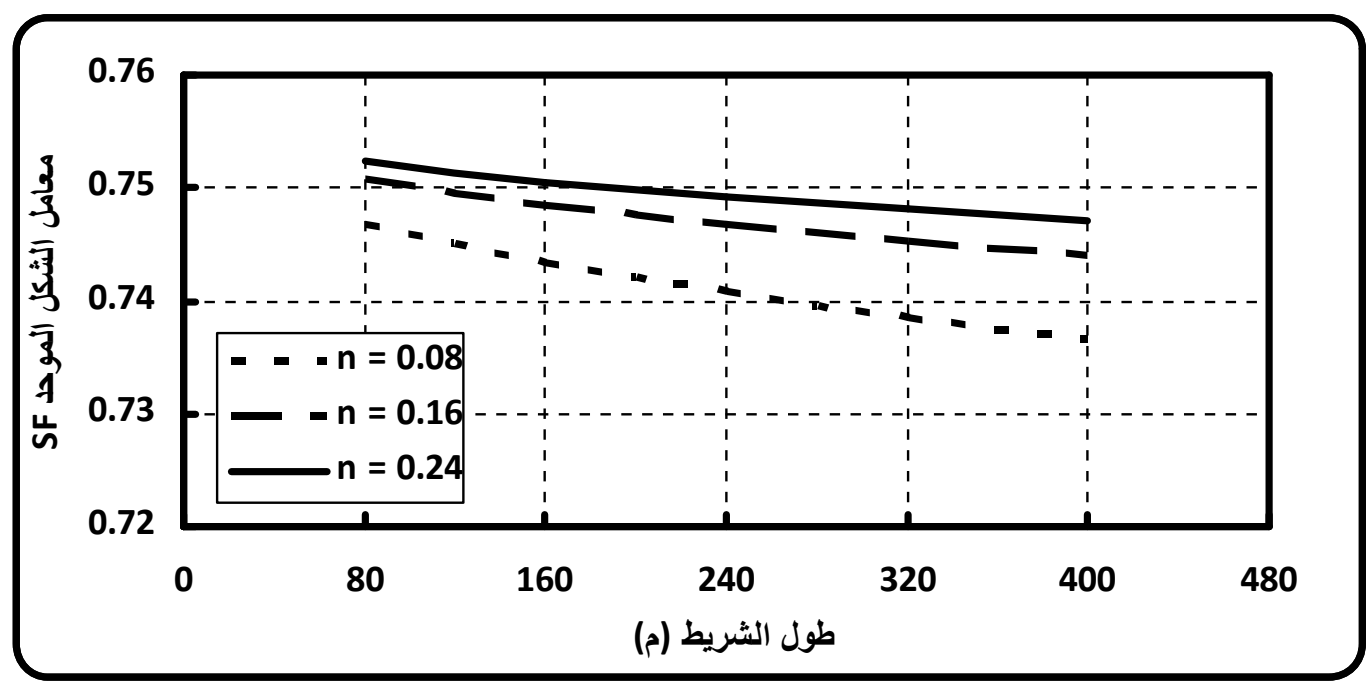

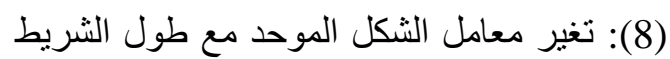

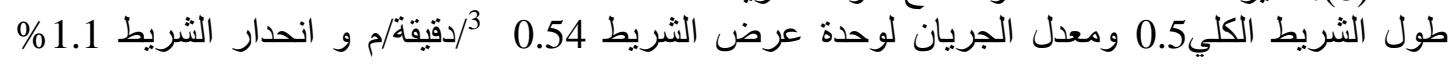
0.543

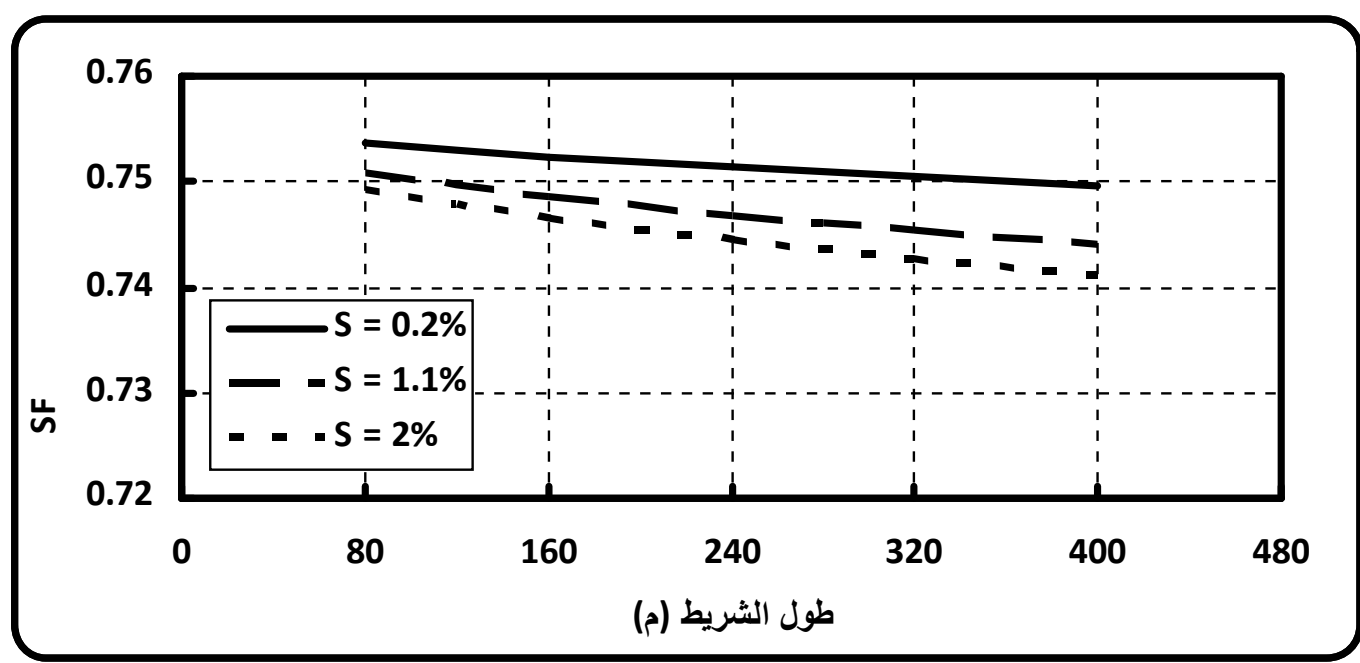

بط في حالة

(9) (9غير معامل الثكل الموحد مع طول الثريط 0.16 ومعدل الجريان لوحدة عرض الثريط 0.54 ُدقيقة/م واس دالة الارتثاح

0.5 الثريط الكلي

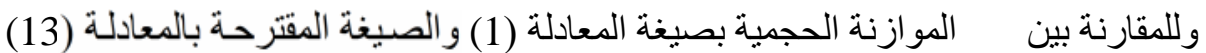

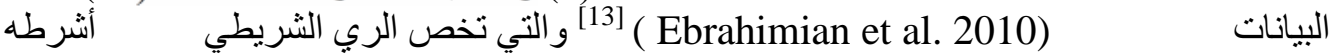

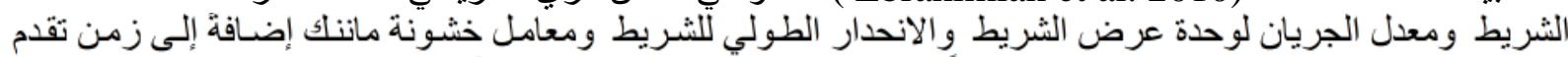

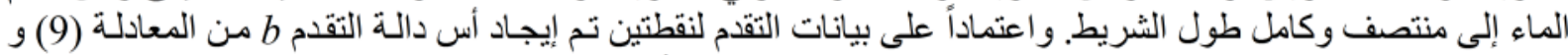

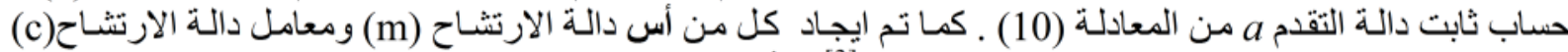

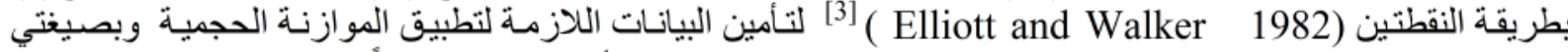
المعادلات (1) و (13). و هذه البيانات معروضة في الجدول(2) و ولثلاثة أشرطه. و اعتماداً على هذه البيانات تم ايجاد $\mathrm{x}$

(2) (20) الييانات المنشورة في (Ebrajhimian etal.2010) 


\begin{tabular}{|c|c|c|c|}
\hline الشريط & الشريط & الثريط الاول & \\
\hline 170 & 170 & 180 & طول الثريط (م) \\
\hline 3.02 & 3.10 & 1.66 & معدل الجريان(لتز/ثا/م) \\
\hline 0.0066 & 0.007 & 0.0082 & ( / ) \\
\hline 0.02 & 0.04 & 0.04 & \\
\hline 28.7 & 35.7 & 45.7 & الشريط (دقيقة) \\
\hline 80 & 107 & 175.1 & لماء لكامل طول الشريطة \\
\hline 0.676 & 0.631 & 0.516 & (b) \\
\hline 8.783 & 8.891 & 12.523 & (a) \\
\hline 0.427 & 0.477 & 0.789 & (m) \\
\hline 13.772 & 13.690 & 1.718 & (c) \\
\hline
\end{tabular}

(12-10) تغير مسافة تقدم الماء زمن تقدم المـاء

. [ $\left.q^{*} t_{x}=0.75^{*} X^{*}\left(d_{0}+D_{0}\right), q^{*} t_{x}=\sigma_{y}^{*} X^{*} d_{0}+\sigma_{z}^{*} D_{0}{ }^{*} x\right]$ (13)
(13)

ة وذللك بصيغة كل من المعادلتين (1) بتطبيق كل من المعادلتين (1) تبين من هده

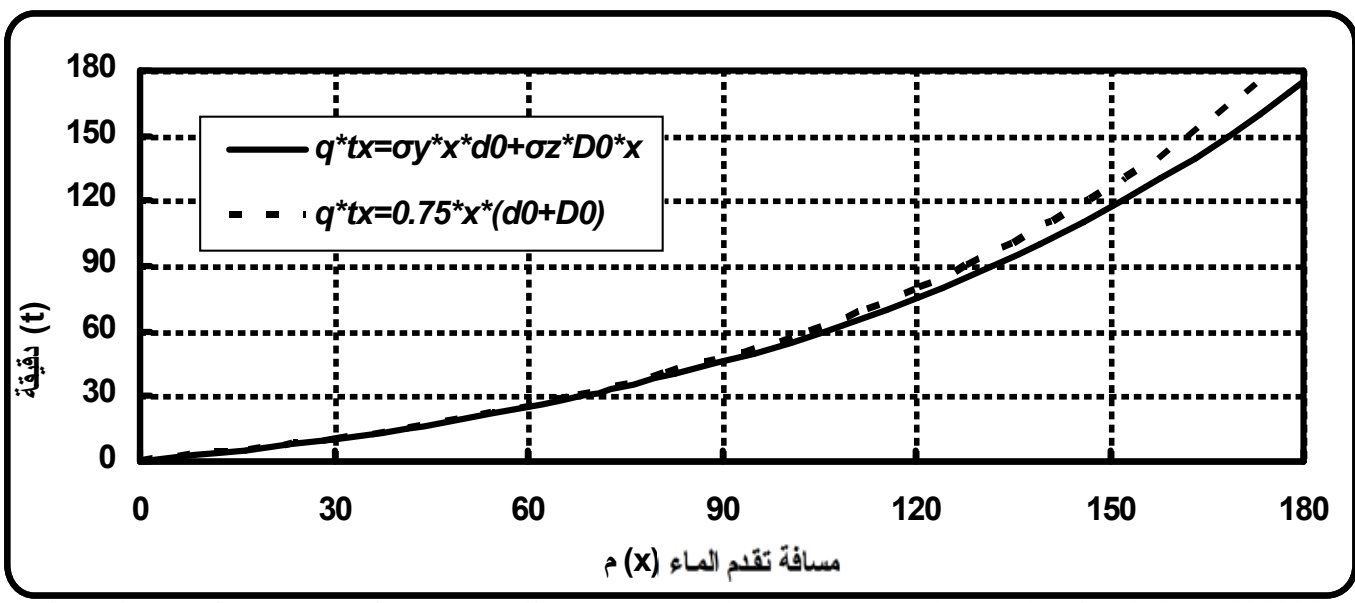

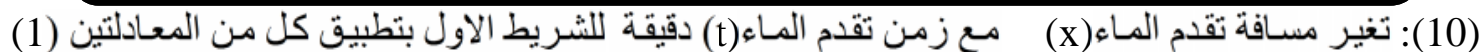
. [ $q^{*} t_{x}=0.75^{*} X^{*}\left(d_{0}+D_{0}\right), q^{*} t_{x}=\sigma_{y}^{*} x^{*} d_{0}+\sigma_{z}^{*} D_{0}^{*} x$

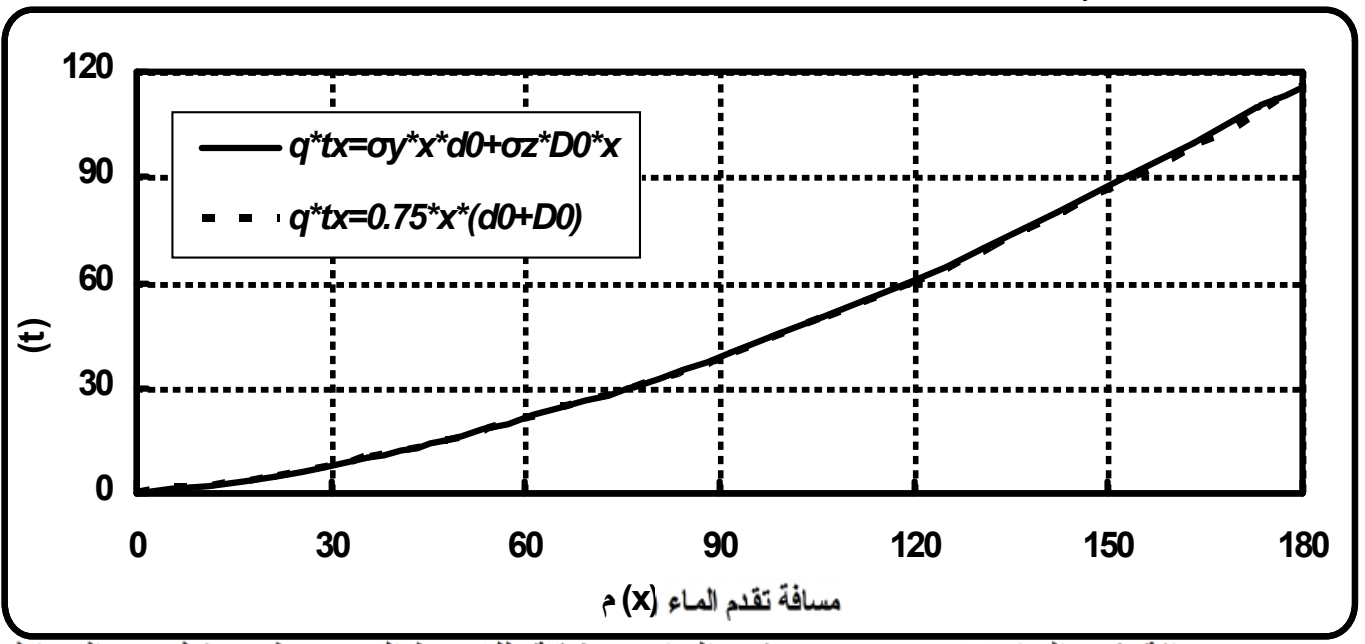

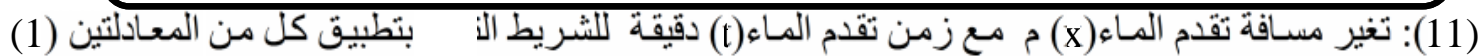
$\left[q^{*} t_{X}=0.75^{*} X^{*}\left(d_{0}+D_{0}\right), q^{*} t_{X}=\sigma_{y}^{*} X^{*} d_{0}+\sigma_{z}^{*} D_{0}^{*} x\right]$ 


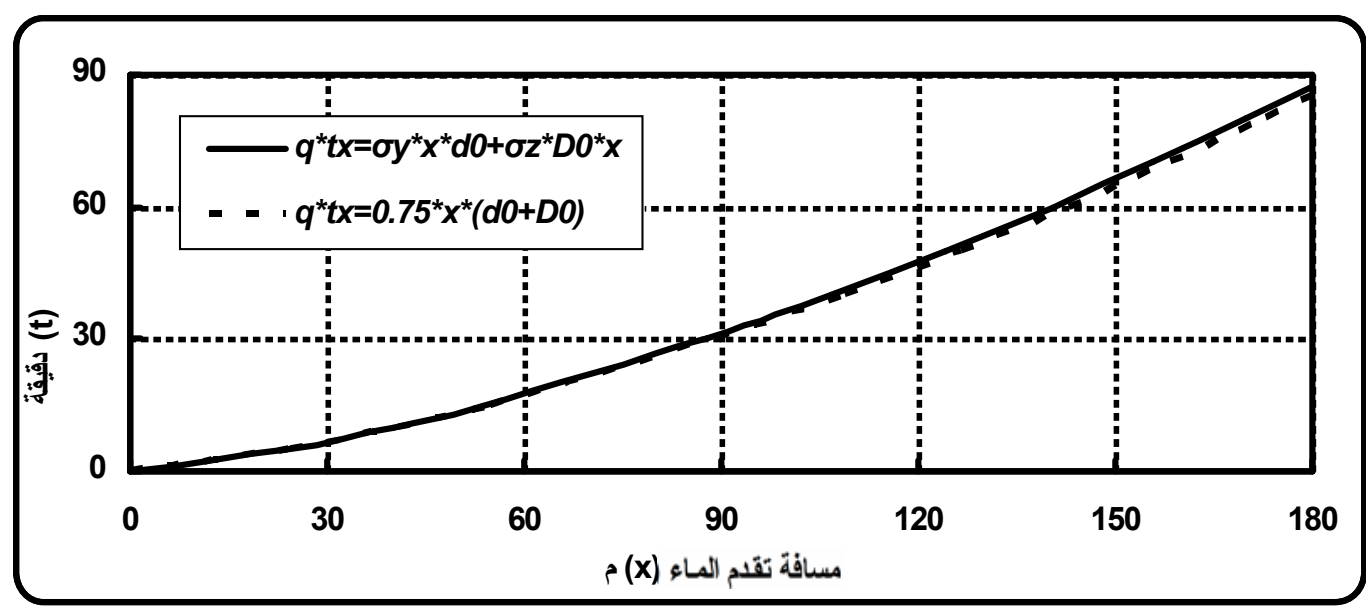

بتطبيق كل من المعادلتين (1)

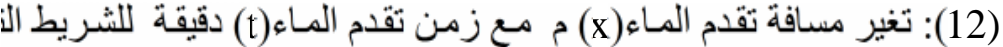
. [ $\left.q^{*} t_{x}=0.75^{*} X^{*}\left(d_{0}+D_{0}\right), q^{*} t_{x}=\sigma_{y}^{*} X^{*} d_{0}+\sigma_{z}^{*} D_{0}^{*} x\right]$

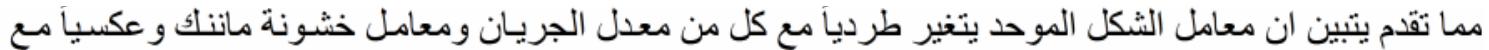

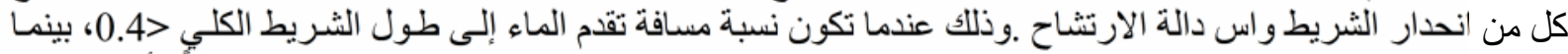

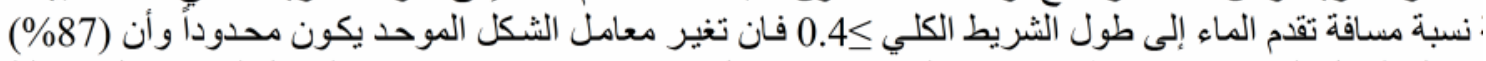

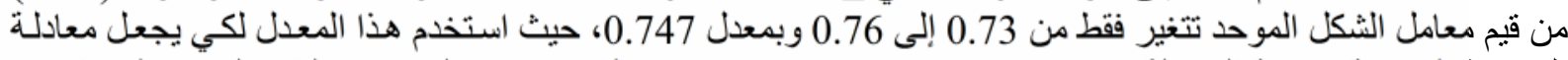
الموازنة الحجمية بصيغة المعادلة (13) [ (13) تطابق يكاد يكون تماماً بالمقارنة مع معادلة الموازنة الحجمية بصيغة المعادلة (1) [ . 1(

1. م، احمد يوسف وحقي إسماعيل ياسين، (1992). "هندسـة نظم الري الحقلي"، دار الكتب للطباعة و النشر / جامعة

2. Christiansen JE, Bishop AA, Kiefer FW Jr, Fok Yu-Si (1966) Evaluation of intake rate constants as related to advance ofwater in surface irrigation. Trans ASAE 9: 671 \pm 674

3. Elliott, R. L., and Walker, W. R., (1982). "Field Evaluation of Furrow Infiltration and Advance Functions". Trans. ASAE. 25】(2), 396-400. Engineering, ASCE. 123(4): 300306.

4. Walker, W.R., Skogerboe, G.V., (1987)." Surface Irrigation. Theory and Practice'. PrenticeHall Inc., Englewood Cliffs, New Jersey, p. 386.

5. Fok, Y. S., and Bishop, A. A. (1965). "Analysis of Water Advance in Surface Irrigation." Journal of Irrigation and Drainage Engineering, ASCE. 91(1), 99-116.

6. Valiantzas, J.D., (1997a). "Surface Irrigation Advance Equation: Variation of Subsurface Shape Factor". Journal of Irrigation and Drainage

7. Valiantzas, J.D., (1997b). "Volume Balance Irrigation Advance Equation: Variation of Surface Shape Factor". Journal of Irrigation and Drainage Engineering, ASCE. 123(4): 307-402.

8. Alazba, A. A. (1999). "Explicit Volume Balance Model Solution". Journal of Irrigation and Drainage Engineering, ASCE. 125(5), 273-279.

9. Theodor, S. S., A. J. Clemmens and E. Bautista (2011). " Shape Factors For Elements Of The Infiltration Profile In Surface Irrigation -A Generic Approach ". Journal of Irrigation and Drainage Engineering. Submitted September 29, 2010, accepted August 24, 2011. 
10. Weibo, N., F. Liangjun and M. Xiaoyi, (2012)." Estimated Infiltration Parameters and Manning Roughness in Border Irrigation".2011 John Wiley \& Sons, Ltd Irrig. and Drain. 61: 231-239.

11. ياسين، حقي إسماعيل،ونو ال محمد ججو ، (1997). "تحليل طور تقدم المـاء في الري الثريطي". مجلة هندسة

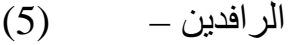

12. أمين، احمد علي محمد، (2010). "تقويم دو ال التقدم و التثرب في الري بالمروز". رسالة ماجستير ، قسم هندسة الموارد المائية/ كلية الهنسة/جامعة الموصل.

13. Ebrahimian,H. , A. Liaghat ,B. G.Alavijeh and F. Abbasi.(2010)" Evaluation of various quick methods for estimating furrow and border infiltration parameters" Irrig Sci 28:479488 .

تم اجراء البحث في كلية ألهندسة = جامعة ألموصل 